\title{
The $-2518 \mathrm{~A} / \mathrm{G}$ Polymorphism in the $M C P-1$ Gene and Tuberculosis Risk: A Meta-Analysis
}

\author{
Yonggang Zhang ${ }^{1}$, Jie Zhang ${ }^{2}$, Lingjun Zeng ${ }^{3}$, Honglang Huang ${ }^{1,4}$, Min Yang ${ }^{1,5}$, Xiaowei Fu' ${ }^{1}$, Can Tian ${ }^{1}$, \\ Zhangpeng Xiang ${ }^{4}$, Jin Huang ${ }^{4 *}$, Hong Fan ${ }^{1 *}$
}

1 Department of Respiratory Medicine, West China Hospital of Sichuan University, Chengdu, Sichuan, China, 2 Key Laboratory of Laboratory Medicine, Ministry of Education, Zhejiang Provincial Key Laboratory of Medical Genetics, Wenzhou Medical College, Wenzhou, Zhejiang, China, 3 Department of Respiratory Medicine, The First Hospital of Ziyang City, Ziyang, Sichuan, China, $\mathbf{4}$ West China Medical School, Sichuan University, Chengdu, Sichuan, China, 5 Department of Intensive Care Unit, The Second Hospital of Anhui Medical University, Hefei, Anhui, China

\begin{abstract}
Background: The $-2518 \mathrm{~A} / \mathrm{G}$ polymorphism in the monocyte chemoattractant protein-1 (MCP-1) gene has been implicated in the susceptibility to tuberculosis (TB), but the results are not conclusive. The aim of this study is to investigate the association between the $-2518 \mathrm{~A} / \mathrm{G}$ polymorphism in the $M C P-1$ gene and the risk of tuberculosis by meta-analysis.

Methods: We searched Pubmed, Embase, CNKI and Wanfang databases, covering all studies until April 29 ${ }^{\text {th }}, 2011$. Statistical analyses were performed using the Revman4.2 and STATA10.0 software.

Results: A total of 5341 cases and 6075 controls in 13 case-control studies were included in the meta-analysis. The results indicated that the GG homozygote carriers had a $67 \%$ increased risk of TB compared with the A allele carriers (GG vs. $\mathrm{GA}+\mathrm{AA}: \mathrm{OR}=1.67,95 \% \mathrm{Cl}=1.25-2.23, \mathrm{P}=0.0006)$. In the subgroup analysis by ethnicity, significant elevated risks were found in Asians and Latinos, but not in Africans (GG vs. GA+AA: OR=1.79, 95\%Cl=1.19-2.70 and $P=0.005$ for Asians; OR=2.15, $95 \% \mathrm{Cl}=1.32-3.51$ and $\mathrm{P}=0.002$ for Latinos; $\mathrm{OR}=1.28,95 \% \mathrm{Cl}=0.45-3.64$ and $\mathrm{P}=0.65$ for Africans).

Conclusion: This meta-analysis suggested that the $-2518 \mathrm{~A} / \mathrm{G}$ polymorphism of $M C P-1$ gene would be a risk factor for TB in Asians and Latinos, while not in Africans.
\end{abstract}

Citation: Zhang Y, Zhang J, Zeng L, Huang H, Yang M, et al. (2012) The -2518A/G Polymorphism in the MCP-1 Gene and Tuberculosis Risk: A Meta-Analysis. PLoS ONE 7(7): e38918. doi:10.1371/journal.pone.0038918

Editor: Samithamby Jeyaseelan, Louisiana State University, United States of America

Received July 20, 2011; Accepted May 15, 2012; Published July 30, 2012

Copyright: (c) 2012 Zhang et al. This is an open-access article distributed under the terms of the Creative Commons Attribution License, which permits unrestricted use, distribution, and reproduction in any medium, provided the original author and source are credited.

Funding: This study was supported by grants \#30470761 and 30871117 from the National Natural Science Foundation of China and grant \#2012JY0013 from the Science and Technology Department of Sichuan province. The funders had no role in study design, data collection and analysis, decision to publish, or preparation of the manuscript.

Competing Interests: The authors have declared that no competing interests exist.

* E-mail: z85445417@yahoo.cn (HF); michael.huangjin@gmail.com (JH)

\section{Introduction}

Tuberculosis $(\mathrm{TB})$ is still one of the main causes of death due to infectious diseases [1,2]. WHO estimated that there were almost 9.4 million new and relapse TB cases worldwide in 2009 [3]. It is reported that one third of the population had ever been infected by Mycobacterium Tuberculosis(MTB), but only $10 \%$ of the infected population might develop to TB with clinical symptoms [4]. Both the MTB and the host immune response may play important roles in the development of TB [5]. Numerous studies have been performed on the association of genetic variants with TB susceptibility $[4,6,7,8,9]$ and among them, the monocyte chemoattractant protein-1 (MCP-1) gene has been highlighted $[9,10,11,12,13,14,15,16,17,18,19,20]$.

The human MCP-1 gene is located on chromosome 17 (17q11.2). It encodes a protein of 76 amino acids and is $13 \mathrm{kd}$ in size [21]. The MCP-1 is a member of the $\mathrm{C}-\mathrm{C}$ chemokine family. It is a potent chemotactic factor for monocytes $[11,13,14]$, which plays critical roles in the recruitment of macrophages and $\mathrm{T}$ lymphocytes for controlling the dissemination of MTB $[18,19]$. High levels of MCP-1 were also detected in bronchoalveolar lavage fluid of TB patients $[18,19]$. There were many polymorphisms in the region of the $M C P-1$ gene, and among them one important polymorphism named -2518A/G (rs1024611) was widely studied [11]. This polymorphism is located on the promoter region of the $M C P-1$ gene and may modulate the expression level of MCP-1. Compared with the $-2518 \mathrm{~A}$ allele, the MCP-1 -2518G allele was associated with increased production of both transcription and protein translation [11,12,18]. Several studies have reported the association between the $-2518 \mathrm{~A} / \mathrm{G}$ polymorphism with TB risk $[9,10,11,12,13,14,15,16,17,18,19,20]$, but the results were inconclusive. Some studies reported that the polymorphism was associated with increased risk of TB in Mexicans, Koreans, Chinese and Peruvians [14,15,18], while the others reported different results, such as in South African Coloreds, Indians, and Ghanaians [10,16,17]. Meta-analysis is a useful method for investigating the risk factors of genetic diseases, because it uses a quantitative approach to combine the results from different studies with the same topic, and can provide more reliable conclusions [22,23]. Previously, Thye [17] performed a case-control study and meta-analysis to assess the association 


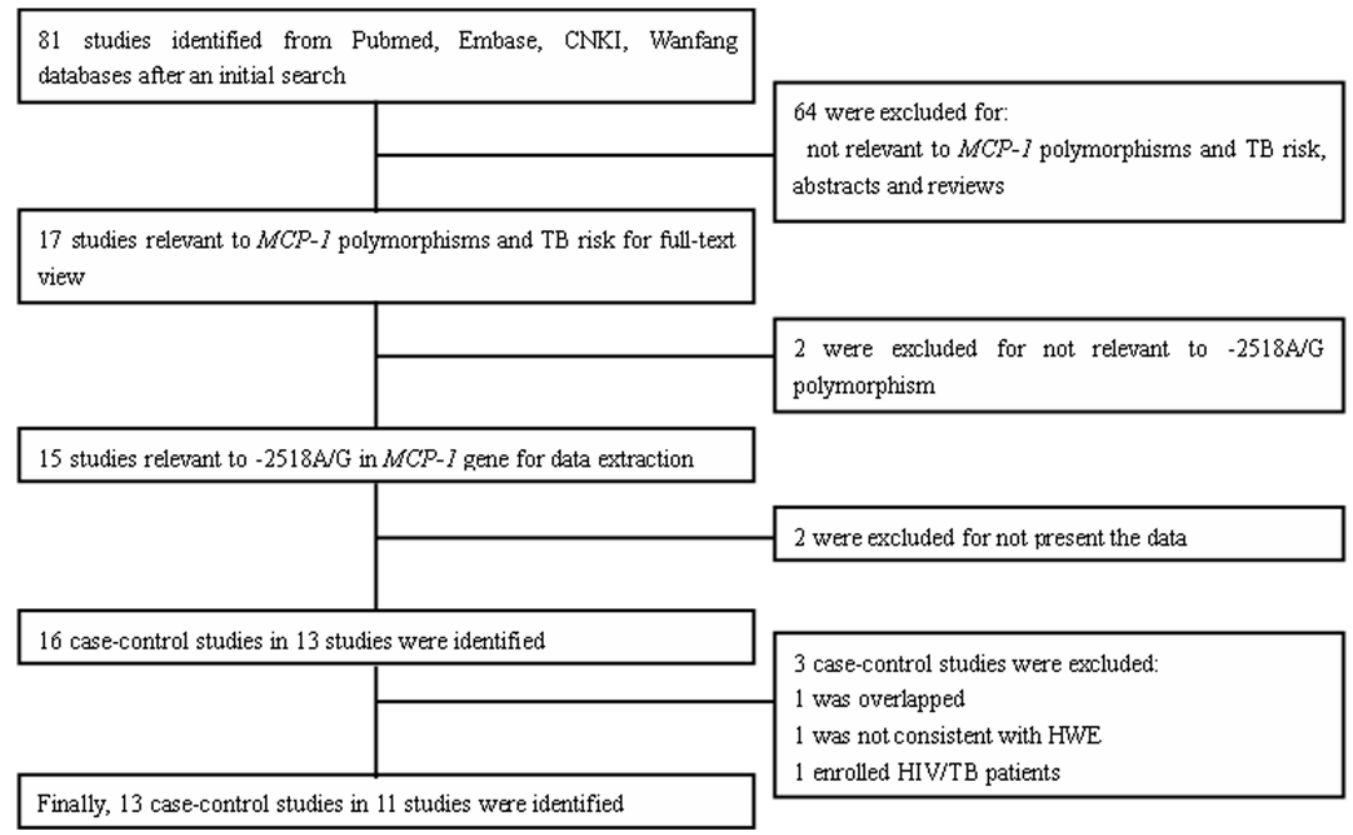

Figure 1. Flow diagram of included/excluded studies. doi:10.1371/journal.pone.0038918.g001

between the risk of $\mathrm{TB}$ and the $-2518 \mathrm{~A} / \mathrm{G}$ polymorphism, but no association was found. However, only five case-control studies were included, and they might be too underpowered to identify reliable conclusions. In addition, the limitation of included studies might also restrict their subgroup analyses of the original ethnic differences. More recent studies concerning the association between the polymorphism and TB risk in different populations have not been included $[9,11,15,18,19,20]$. Furthermore, several important factors which may bias the results of genetic association studies were not clearly addressed, such as, Hardy-Weinberg equilibrium (HWE). The stability of meta-analysis was not discussed and different genotype comparative results were also not mentioned. Thus, we carried out a meta-analysis that included the most updated data to investigate the association between the 2518A/G polymorphism in the MCP-1 gene and the risk of TB, and the ethnic differences. This is, to our knowledge, the most comprehensive meta-analysis regarding the $-2518 \mathrm{~A} / \mathrm{G}$ polymorphism and TB risk.

\section{Materials and Methods}

\section{Study identification and selection}

A systematic literature search in Pubmed database, Embase database, Wanfang database (www.wanfangdata.com.cn) and GNKI database (China National Knowledge Infrastructure, www.cnki.net) were carried out to identify studies involving the association between TB risk and $M C P-1$ polymorphisms on April $29^{\text {th }}, 2011$. The search terms were as follows: 'TB or tuberculosis' in combination with 'polymorphism or variant or mutation' and in combination with 'Monocyte chemoattractant protein-1 or MCP1 or CAL2 or Chemokine (C-C motif) ligand 2'. The languages were limited to English and Chinese. Inclusion criteria were defined as follows: (a) studies evaluated the association between MCP-1 -2518A/G and TB risk; (b) the design had to be a casecontrol study based on unrelated individuals; (c) sufficient data (genotype distributions for cases and controls) was available to estimate an odds ratio (OR) with its 95\%CI; (d) genotype distributions in control group should be consistent with HWE. Studies were excluded if one of the following existed: (a) the design based on family or sibling pairs, (b) the genotype frequencies or number not reported, (c) reviews and abstracts, (d) studies with HIV/TB patients. If more than one study by the same authors using the same case series was published, either the studies with the largest sample size or the ones that were published most recently were included. The supporting PRISMA checklist is available as supporting information; see Checklist S1.

\section{Data extraction}

Two reviewers collected the data and reached a consensus on all items. The following items were extracted from each study if available: first author, year of publication, country of origin, ethnicity, sample size, TB definition, genotyping method, and genotype number in cases and controls.

\section{Statistical analysis}

The strength of the association between the MCP-1 -2518A/G polymorphism and the risk of TB was measured by OR and $95 \%$ CI. The recessive genetic model (GG vs. AG+AA) was used to evaluate the pooled OR. OR was analyzed by fixed-effects model or random-effects model according to the results of heterogeneity [24]. Heterogeneity was evaluated by a $X^{2}$ based $Q$ statistic and was considered statistically significant when $P<0.10$. When the $P$ value was $>0.10$, the pooled OR was calculated by the fixedeffects model, otherwise, the random-effects model was used. The significance of the pooled OR was determined by the Z-test and was considered statistically when the $P$ value was less than 0.05 . To evaluate the ethnicity-specific effects, subgroup analyses by ethnic groups were performed. For this polymorphism, other genetic models (GG+GA vs. AA and G vs. A) were also used to assess the association with the risk of TB.

Publication bias was analyzed by Begg's funnel plots and Egger's test [24]. Sensitivity analysis was performed by sequentially excluding individual study to assess the stability of the results [24]. HWE was tested by Pearson's $X^{2}$ test $(\mathrm{P}<0.05$ means 


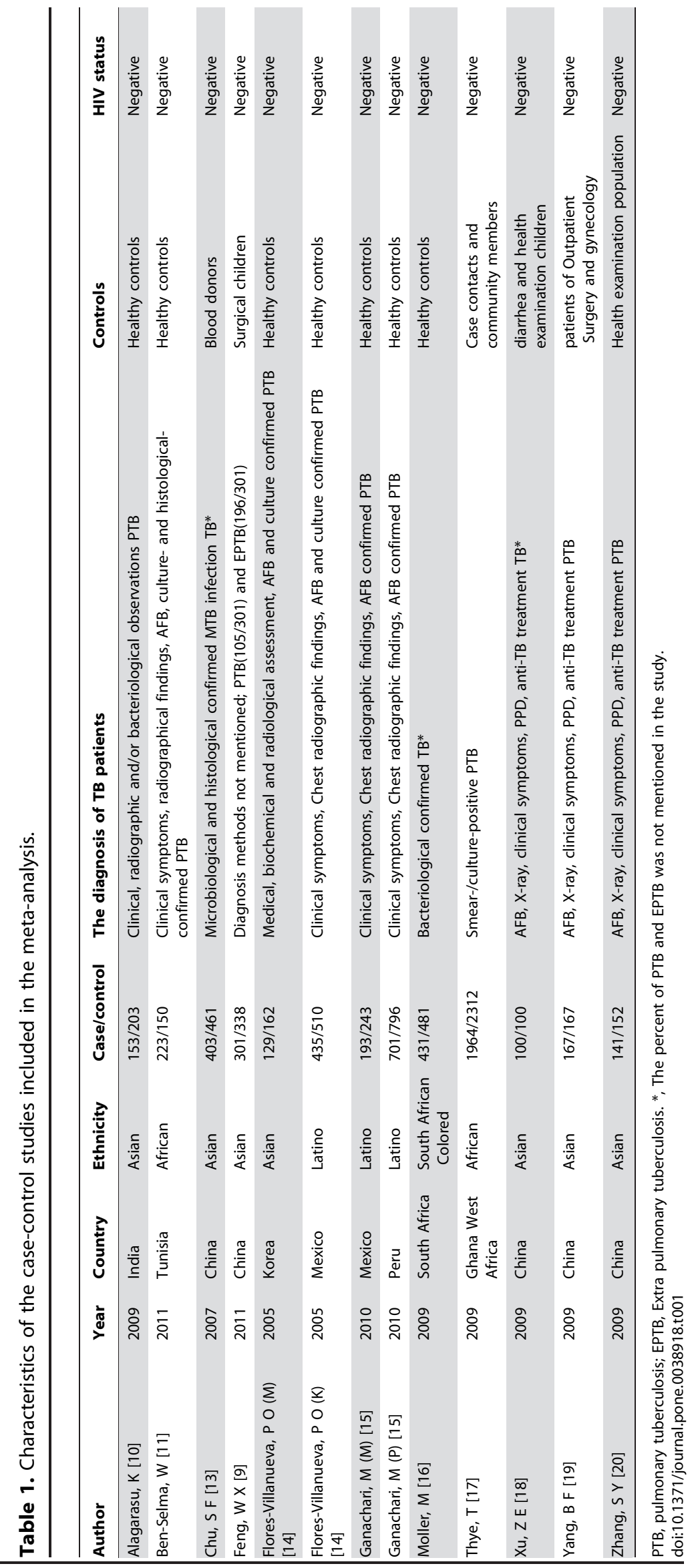


Table 2. Distributions of $M C P-1$ genotype and allele among TB patients and controls.

\begin{tabular}{|c|c|c|c|c|c|c|c|c|c|c|c|}
\hline \multirow[t]{2}{*}{ Author } & \multicolumn{3}{|l|}{ Case } & \multicolumn{3}{|c|}{ Control } & \multicolumn{2}{|l|}{ Case } & \multicolumn{2}{|c|}{ Control } & \multirow{2}{*}{$\frac{\text { HWE }}{P \text { value }}$} \\
\hline & AA & GA & GG & AA & GA & GG & A & G & A & G & \\
\hline Alagarasu, K [10] & 78 & 54 & 21 & 93 & 81 & 29 & 210 & 96 & 267 & 139 & 0.105 \\
\hline Ben-Selma, W [11] & 110 & 87 & 26 & 93 & 49 & 8 & 307 & 139 & 235 & 65 & 0.645 \\
\hline Chu, S F [13] & 93 & 200 & 110 & 115 & 233 & 113 & 386 & 420 & 463 & 459 & 0.816 \\
\hline Feng, W X [9] & 38 & 157 & 106 & 51 & 170 & 117 & 233 & 369 & 272 & 404 & 0.400 \\
\hline Flores-Villanueva, P O (K)*[14] & 20 & 63 & 46 & 66 & 74 & 22 & 103 & 155 & 206 & 118 & 0.862 \\
\hline Flores-Villanueva, P O (M)*[14] & 38 & 168 & 229 & 124 & 249 & 137 & 244 & 626 & 497 & 523 & 0.605 \\
\hline Ganachari, M (M)**[15] & 23 & 77 & 93 & 46 & 127 & 70 & 123 & 263 & 219 & 267 & 0.386 \\
\hline Ganachari, M $(P)^{* *}[15]$ & 74 & 273 & 354 & 98 & 371 & 327 & 421 & 981 & 567 & 1025 & 0.646 \\
\hline Moller, M [16] & 263 & 142 & 26 & 270 & 173 & 38 & 668 & 194 & 713 & 249 & 0.170 \\
\hline Thye, T [17] & 1355 & 546 & 63 & 1472 & 748 & 92 & 3256 & 672 & 3692 & 932 & 0.803 \\
\hline$X u, Z$ E [18] & 15 & 49 & 36 & 41 & 45 & 14 & 79 & 121 & 127 & 73 & 0.770 \\
\hline Yang, B F [19] & 21 & 62 & 84 & 42 & 83 & 42 & 104 & 230 & 167 & 167 & 0.938 \\
\hline Zhang, S Y [20] & 16 & 76 & 49 & 40 & 77 & 35 & 108 & 174 & 157 & 147 & 0.860 \\
\hline
\end{tabular}

*, K, Korea, M, Mexico; ${ }^{* *}, \mathrm{M}$, Mexico, P, Peru.

doi:10.1371/journal.pone.0038918.t002

deviated from HWE). All statistical tests were performed using the Revman4.2 and STATA 10.0 software.

\section{Results}

\section{Studies selection process and characteristics}

As it shows in Figure 1, a total of 81 results were identified after an initial search. After reading the titles and abstracts, 17 potential studies were included for full-text view. After reading full texts, two studies were excluded for not being relevant to TB risk and the MCP-1 gene. Thus, 15 studies were left for data extraction. In addition, two studies were excluded for not reporting the usable data. Three studies reported two cohorts each [14,15,17] and each cohort was extracted as a separate case-control study. Thus, a total of 16 case-control studies in 13 studies were identified. The genotype in the control group for one case-control study was not consistent with HWE and that study was excluded. One casecontrol study was excluded for data overlapping or duplicated data, and one study was excluded for enrolling the HIV patients. Thus, a total of 13 case-control studies in 11 studies were identified $[9,10,11,13,14,15,16,17,18,19,20]$. Seven case-control studies were performed with Asians $[9,10,13,14,18,19,20]$, two with Africans [11,17], three with Latinos [14,15] and one with South African Coloureds (a mixed ancestry which includes San, Khoi, Malaysians, African black and Europeans) [16]. The characteristics of each case-control study are listed in Table 1. Genotype and allele distributions for each case-control study are shown in Table 2.

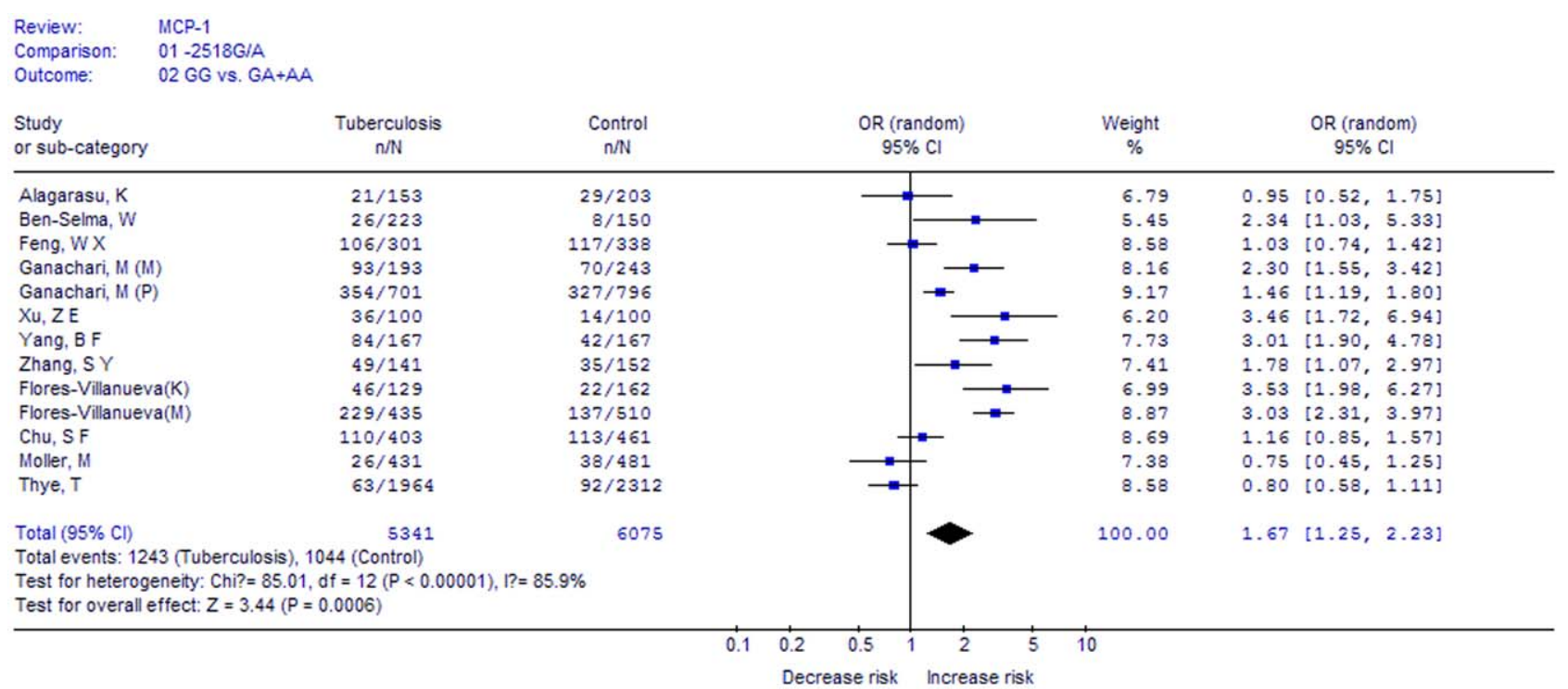

Figure 2. Meta-analysis with a random-effects model for the association between TB risk and the $M C P-1-2518 A / G$ polymorphism (GG vs. AA+GA).

doi:10.1371/journal.pone.0038918.g002 


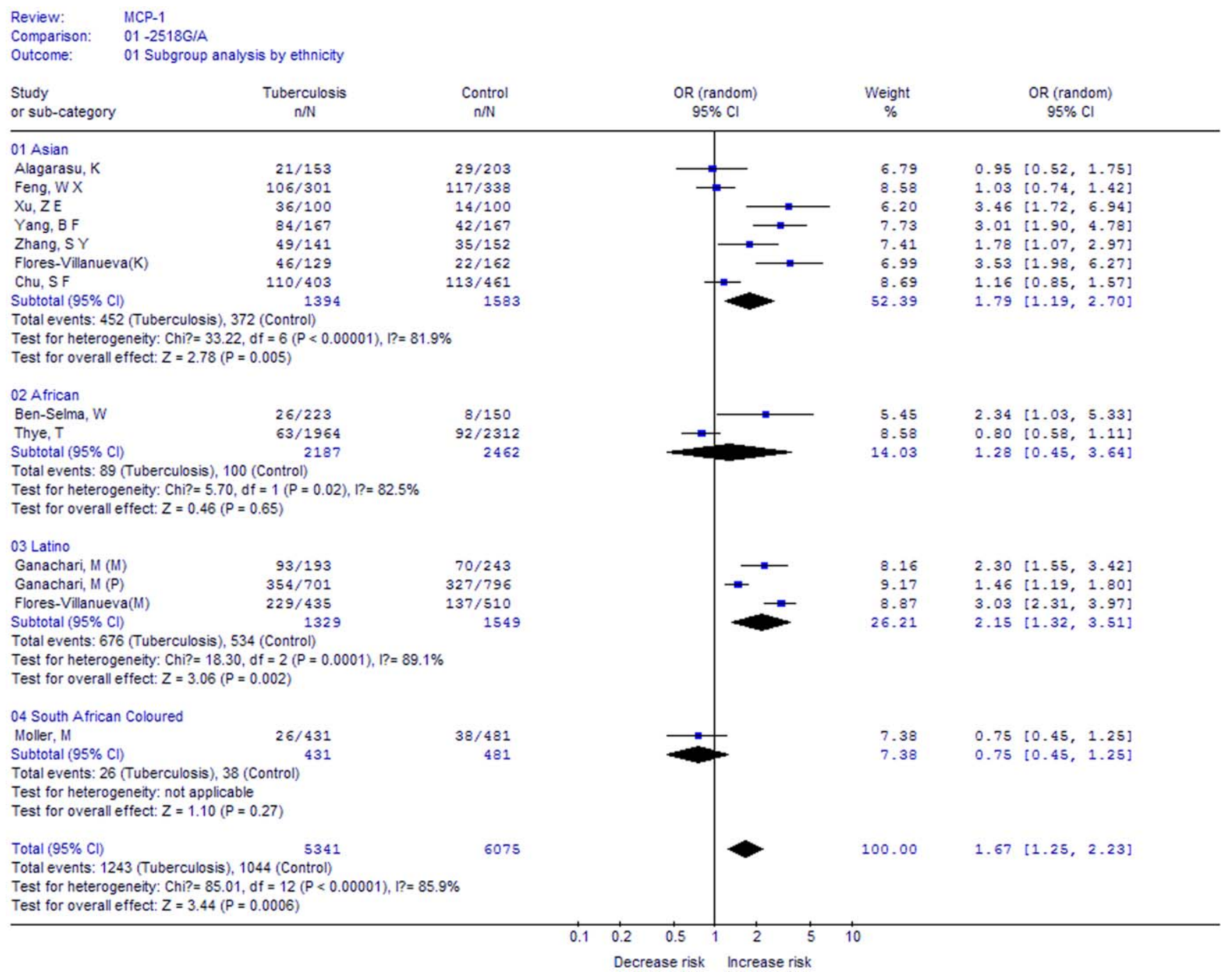

Figure 3. Meta-analysis with a random-effects model for the association between TB risk and the $M C P-1-2518 A / G$ polymorphism (GG vs. AA+GA): subgroup analysis by ethnicity.

doi:10.1371/journal.pone.0038918.g003

\section{Quantitative data synthesis}

A total of 5341 cases and 6075 controls in 13 case-control studies were included. We analyzed the heterogeneity of GG vs. GA+AA for all 13 studies and the value of $X^{2}$ was 85.01 with 12 degrees of freedom and $\mathrm{P}<0.00001$. Thus, we chose the randomeffects model to synthesize the data. Overall, OR was 1.67 $(95 \% \mathrm{CI}=1.25-2.23)$ and the test for overall effect $\mathrm{Z}$ value was $3.44(\mathrm{P}=0.0006)$ for $\mathrm{GG}$ vs. GA+AA model (Figure 2). The results suggested that individuals who carry the GG homozygote may have a $67 \%$ increased risk of $\mathrm{TB}$ compared with the $\mathrm{A}$ allele carriers $(\mathrm{AA}+\mathrm{AG})$.

In the subgroup analysis by ethnicity (GG vs. GA+AA, Figure 3), significantly increased risks were found among Asians (OR, 1.79; 95\%CI, 1.19-2.70; $P=0.005$ ) and Latinos (OR, 2.15; 95\%CI, $1.32-3.51 ; P=0.002)$, but not for Africans (OR, 1.28; 95\%CI, $0.45-3.64 ; \quad P=0.65)$. Thus, the results indicated that the polymorphism is associated with increased risks of TB in Asians and Latinos, but not in Africans.

A summary of results from other comparisons is listed in Table 3.

\section{Publication bias}

Publication bias was analyzed by using the Begg's funnel plots and Egger's test. The shape of the funnel plots was seemed symmetrical in the GG vs. GA+AA comparison genetic model, suggesting the absence of publication bias (Figure 4). The Egger's test was performed to provide statistical evidence of funnel plot asymmetry. The results indicated a lack of publication bias $(t=0.56, P=0.584)$.

\section{Sensitivity analysis}

After sequentially excluding each case-control study, statistically similar results were obtained for GG vs. GA+AA (all P values were $<0.05$ ), suggesting the stability of this meta-analysis (data not shown).

\section{Discussion}

It is well known that TB is a complex infectious disease that involves the infection of MTB, host immune response and geneenvironment interactions. There is different individual susceptibility to TB reported. Host genetic factors, including variants of 
Table 3. Summary of results from different comparative genetic models.

\begin{tabular}{|c|c|c|c|c|c|c|c|c|}
\hline \multirow[t]{2}{*}{$-2518 A / G$} & \multirow[t]{2}{*}{$\mathbf{N}^{\mathrm{a}}$} & \multirow[t]{2}{*}{ Cases/Controls } & \multicolumn{2}{|l|}{ GG vs. GA+AA } & \multicolumn{2}{|l|}{ GG+GA vs. AA } & \multicolumn{2}{|l|}{ G vs. A } \\
\hline & & & OR(95\%Cl) & $P^{b}$ & OR(95\%Cl) & $P^{b}$ & OR(95\%Cl) & $\mathbf{P}^{\mathbf{b}}$ \\
\hline Total & 13 & $5341 / 6075$ & $1.67(1.25,2.23)$ & 0.0006 & $1.60(1.17,2.18)$ & 0.003 & $1.46(1.15,1.86)$ & 0.002 \\
\hline Asian & 7 & $1394 / 1583$ & $1.79(1.19,2.70)$ & 0.005 & $1.87(1.18,2.95)$ & 0.007 & $1.58(1.14,2.18)$ & 0.005 \\
\hline Afrian & 2 & $2187 / 2462$ & $1.28(0.45,3.64)$ & 0.65 & $1.12(0.53,2.34)$ & 0.77 & $1.13(0.58,2.24)$ & 0.72 \\
\hline Latino & 3 & $1329 / 1549$ & $2.15(1.32,3.51)$ & 0.002 & $1.90(0.97,3.72)$ & 0.06 & $1.76(1.16,2.68)$ & 0.008 \\
\hline $\begin{array}{l}\text { South African } \\
\text { Colored }\end{array}$ & 1 & $431 / 481$ & $0.75(0.45,1.25)$ & 0.27 & $0.82(0.63,1.06)$ & 0.14 & $0.83(0.67,1.03)$ & 0.09 \\
\hline
\end{tabular}

SAC: South African Coloureds. a, number of case-control studies; b, p value for z-test.

doi:10.1371/journal.pone.0038918.t003

susceptible genes involved in the pathogenesis of $\mathrm{TB}$, may affect these differences $[4,7,25]$. Therefore, more interest focusing on the genetic susceptibility to TB has led to a growing attention in recent years. The $-2518 \mathrm{~A} / \mathrm{G}$ polymorphism in the $M C P-1$ gene is one of the most widely studied polymorphisms among them. A growing number of studies have indicated that this polymorphism is associated with the increased risk of TB. However, the results from different published studies were inconsistent. Thus, we performed this meta-analysis to comprehensively analyze these associations. To our knowledge, this is the largest meta-analysis to date investigating the association between the $M C P-1-2518 \mathrm{~A} / \mathrm{G}$ polymorphism and TB risk.

Compared with the previous case-control study and metaanalysis by Thye [17], our meta-analysis included a total of 13 case-control studies with 5341 cases and 6075 controls, which doubled the cases compared to the study mentioned above. In previous meta-analysis, the authors did not find a significant association, while we found a significant association between this polymorphism and TB risk in our study. There are several reasons that may explain the different results between our study and Thye's study. First, our meta-analysis included more case-control studies than Thye's study, thus our study is more powerful and the conclusion may be more reliable. Second, the studied populations came from more regions with different genetic backgrounds than previous meta-analysis, so our results might be more conclusive. Third, only allelic frequencies were compared in Thye's study, while the comparisons of all genetic models were performed in our study. In total, these results indicate that this polymorphism may

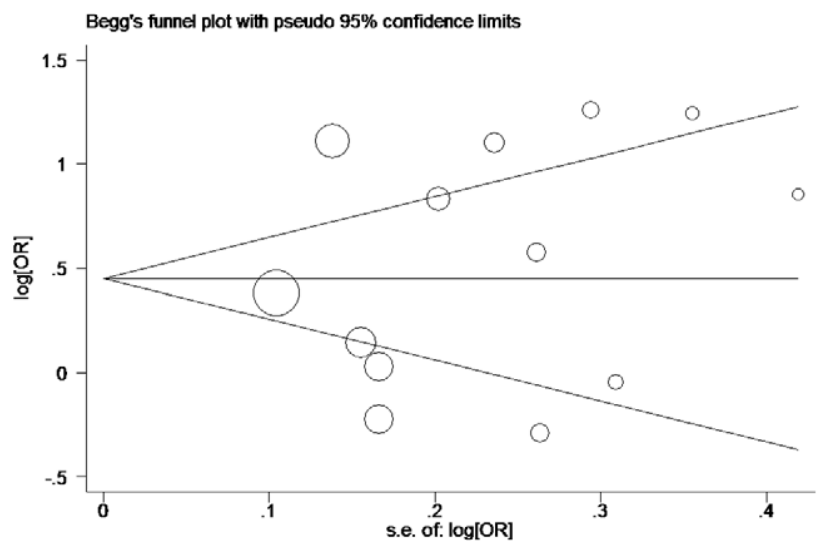

Figure 4. Begg's funnel plot for publication bias in selection of studies on the $M C P-1-2518 A / G$ polymorphism (GG vs. AA+GA). doi:10.1371/journal.pone.0038918.g004 contribute to TB pathogenesis, and help to explain individual differences in host susceptibility to TB.

After subgroup analyses according to ethnicity, we found that the variant GG homozygote carriers had a $79 \%$ increased risk of $\mathrm{TB}$ in Asians and $115 \%$ in Latinos, but not in Africans. It is possible that different genetic backgrounds may account for these differences. In addition, TB is a complex disease that resulted from the infection of MTB and genetic susceptibility. It is possible that these differences in genetic susceptibility might also be owed to individual status or other factors [24]. Thus, further studies are needed to assess the effect of more interactions in different ethnicities and to validate our results.

We must also mention that heterogeneity is important in metaanalysis. In our meta-analysis, significant heterogeneity was found in the overall analysis. There are several factors accounting for heterogeneity. Firstly, the genetic backgrounds for cases and controls may account for it. Different populations have different genetic backgrounds, which contribute to genetic heterogeneity. Secondly, environmental exposures in different case-control studies were not investigated, and these may also influence genetic susceptibility. Thirdly, the cases and controls included were not very homogenous in the meta-analysis: some case-control studies included both pulmonary TB (PTB) patients and extra-pulmonary TB (EPTB) patients and some included PTB only; some studies employed healthy populations as controls, while some studies employed surgical patients as controls. Thus, the results should be considered with caution, and in the future, more studies should be performed to assess these results.

Some limitations should be acknowledged when explaining the results. Firstly, only published data which were included by the selected databases were included; it is possible that some relevant published studies or unpublished studies which had null results were missed, which might bias the results, while our statistical tests may not have totally shown it. Secondly, all case-control studies were from Asians, Africans, Latinos and South African Coloureds; thus, the results may be applicable to these ethnic populations only. Thirdly, data were not stratified by other factors such as gender status and the clear diagnosis methods of TB, because sufficient information could not be extracted from the original studies. Fourth, we should pay attention to the control population, as it is unknown whether the controls had ever been infected by MTB or not, we also could not exclude the possibility that some of the controls were latent TB infection (LTBI) and they might develop active TB in future.

Despite of these limitations, this study also has some advantages. First, it updates the recent data for this polymorphism and TB risk. Second, it is the first time studying the ethnic specificity and $M C P-1-2518 \mathrm{~A} / \mathrm{G}$ polymorphism interactions. 
Third, the methodological issues for meta-analysis, such as, heterogeneity, publication bias, and stability of results were all well investigated.

In conclusion, this meta-analysis suggested that the MCP-1 $2518 \mathrm{~A} / \mathrm{G}$ polymorphism is associated with increased risk of TB, especially in Asians and Latinos. More studies with a larger group of populations should be performed to analyze these associations, especially in Africans and Caucasians.

\section{References}

1. Lawn SD, Zumla AI (2011) Tuberculosis. The Lancet 378: 57-72.

2. Hasan Z, Cliff JM, Dockrell HM, Jamil B, Irfan M, et al. (2009) CCL2 Responses to Mycobacterium tuberculosis Are Associated with Disease Severity in Tuberculosis. PLoS One 4: e8459.

3. WHO (2010) Global tuberculosis control 2010

4. KIM JUS (2011) Influence Of Vitamin D Receptor Polymorphism On Tuberculosis Among South Korean. American Journal of Respiratory and Critical Care Medicine 183: A3346.

5. Mokrousov I, Wu XR, Vyazovaya A, Feng WX, Sun L, et al. (2011) Polymorphism of $3^{\prime}$ UTR Region of TNFR2 Coding Gene and its Role in Clinical Tuberculosis in Han Chinese Pediatric Population. Infection, Genetics and Evolution.

6. Arce-Mendoza AY, Rosas-Taraco AG, Revol A, Salinas-Carmona MC, Rendon A, et al. (2007) CD14 C(-159) T polymorphism is a risk factor for development of pulmonary tuberculosis. Journal of Infectious Diseases 196: 1698-1706.

7. Ben-Selma W, Ben-Kahla I, Boukadida J, Harizi H (2011) Contribution of P2X7 1513A/C loss-of-function polymorphism to extrapulmonary tuberculosis susceptibility in Tunisian populations. FEMS Immunol Med Microbiol.

8. Cooke GS, Campbell SJ, Sillah J, Gustafson P, Bah B, et al. (2006) Polymorphism within the Interferon-gamma/Receptor complex is associated with pulmonary tuberculosis. American Journal of Respiratory and Critical Care Medicine 174: 339-343.

9. Feng WX, Mokrousov I, Wang BB, Nelson H, Jiao WW, et al. (2011) Tag SNP Polymorphism of CCL2 and Its Role in Clinical Tuberculosis in Han Chinese Pediatric Population. PLoS One 6: e14652.

10. Alagarasu K, Selvaraj P, Swaminathan S, Raghavan S, Narendran G, et al. (2009) CCR2, MCP-1, SDF-1'Á \& DC-SIGN gene polymorphisms in HIV-1 infected patients with \& without tuberculosis. Indian Journal of Medical Research 130: 444-450.

11. Ben-Selma W, Harizi H, Boukadida J (2011) MCP-1- 2518 A/G functional polymorphism is associated with increased susceptibility to active pulmonary tuberculosis in Tunisian patients. Molecular Biology Reports: 1-7.

12. Buijtels PC, van de Sande WW, Parkinson S, Petit PL, van der Sande MA, et al. (2008) Polymorphism in CC-chemokine ligand 2 associated with tuberculosis in Zambia. International Journal of Tuberculosis and Lung Disease 12: 14851488.

13. Chu S, Tam C, Wong H, Kam K, Lau Y, et al. (2007) Association between RANTES functional polymorphisms and tuberculosis in Hong Kong Chinese. Genes and immunity 8: 475-479.

\section{Supporting Information \\ Checklist S1 PRISMA Checklist. \\ (DOG)}

\section{Author Contributions}

Conceived and designed the experiments: HF JH. Performed the experiments: YZ JZ HH. Analyzed the data: LZ MY XF. Contributed reagents/materials/analysis tools: CT ZX. Wrote the paper: YZ JH.

14. Flores-Villanueva PO, Ruiz-Morales JA, Song CH, Flores LM, Jo EK, et al. (2005) A functional promoter polymorphism in monocyte chemoattractant protein̈ 1 is associated with increased susceptibility to pulmonary tuberculosis. The Journal of experimental medicine 202: 1649-1658.

15. Ganachari M, Ruiz-Morales JA, de la Torre JCG, Dinh J, Granados J, et al. (2010) Joint Effect of MCP-1 Genotype GG and MMP-1 Genotype 2G/2G Increases the Likelihood of Developing Pulmonary Tuberculosis in BCGVaccinated Individuals. PLoS One 5: e8881.

16. Moller M, Nebel A, Valentonyte R, van Helden PD, Schreiber S, et al. (2009) Investigation of chromosome 17 candidate genes in susceptibility to TB in a South African population. Tuberculosis 89: 189-194.

17. Thye T, Nejentsev S, Intemann CD, Browne EN, Chinbuah MA, et al. (2009) MCP-1 promoter variant- $362 \mathrm{C}$ associated with protection from pulmonary tuberculosis in Ghana, West Africa. Human Molecular Genetics 18: 381.

18. Xu ZE, Xie YY, Chen JH, Xing LL, Zhang AH, et al. (2009) Monocyte chemotactic protein-1 gene polymorphism and monocyte chemocactic protein-1 expression in Chongqing Han children with tuberculosis Zhonghua Erke Zazhi 47: 200-203.

19. Yang BF, Zhuang B, Li F, ZHANG CZ, Song AO (2009) The relationship between monocyte chemoattactant protein-1 gene polymorphisms and the susceptibility to pulmonary tuberculosis. Zhonghua Jiehe He Huxi Zazhi 32: $454-456$.

20. Zhang SY (2009) Associations between MCP-1 Gene Polymorphisms and Tuberculosis in the Haiyuan Hui People (Chinese).

21. Deshmane SL, Kremlev S, Amini S, Sawaya BE (2009) Monocyte chemoattractant protein-1 (MCP-1): an overview. Journal of Interferon \& Cytokine Research 29: 313-326.

22. Zhang C, Li JP, Lv GQ, Yu XM, Gu YL, et al. (2011) Lack of Association of SULT1A1 R213H Polymorphism with Colorectal Cancer: A Meta-Analysis. PLoS One 6: e19127.

23. Zhang G, Mai R, Huang B (2010) ADH1B Arg47His Polymorphism Is Associated with Esophageal Cancer Risk in High-Incidence Asian Population: Evidence from a Meta-Analysis. PLoS One 5: e13679.

24. Zhang YG, Li XB, Zhang J, Huang J, He C, et al. (2010) The I/D polymorphism of angiotensin-converting enzyme gene and asthma risk: a metaanalysis. Allergy 66: 197-205.

25. Thye T, Scarisbrick G, Browne ENL, Chinbuah MA, Gyapong J, et al. (2009) CTLA4 autoimmunity-associated genotype contributes to severe pulmonary tuberculosis in an african population. PLoS One 4: e6307. 